XII.

Aus dem pharmakologischen Institute der deutschen Universität in Prag.

\title{
Über die Beeinflussung des Purinstoffwechsels durch Phenylcinchoninsäure (Atophan).
}

Von

Dr. Fmil Starkenstein, Assistent am Institute.

Bei Untersuchungen über die Wirkung von Chinolincarbonsäuren und ibren Derivaten fanden $\mathrm{Nicolaier}$ und $\mathrm{Dohrn}{ }^{1}$ ), daB einigen Körpern dieser Reibe die Fähigkeit zukomme, die Harnsäureausscheidung beim Menschen zu steigern. Von den in dieser Richtung untersuchten Substanzen zeigten die genannte Eigenschaft vor allem die Phenylderivate der Chinolincarbonsäuren und unter diesen ganz besonders die 2-Phenylchinolin 4-Carbonsäure, die auch als 2-Phenylcinchoninsäure bezeichnet wird. Sie hat die Formel $\mathrm{C}_{16} \mathrm{H}_{11} \mathrm{NO}_{2}$ und folgende Konstitution.

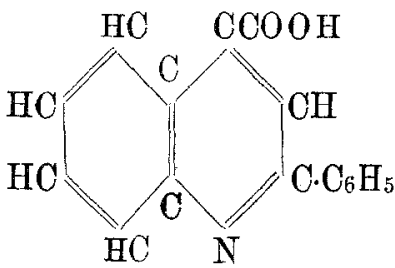

Die Substanz kristallisiert in kleinen farblosen Nadeln (Schmelzpunkt $208-209^{\circ} \mathrm{O}$.), ist in Wasser fast unlöslich, leicht löslich in Alkalien und beim Erwärmen auch in verdünnten Säuren. Sie löst sich ferner leicht in heißem Alkohol, Aceton und siedendem Eisessig, schwer dagegen in Äther und Benzol. Sie ist von bitterem Geschmack.

Nachdem $\mathrm{N}$ i c o I a i e $\mathrm{r}$ und $\mathrm{D}_{3}$ o h r n die relative Ungiftigkeit der Substanz nach Verabreichung per os an Hunden, Kaninchen, an

1) Nicolaier und Dohrn: Über die Wirkung der Chinolincarbonsäuren und ihrer Derivate auf die Ausscheidung der Harnsäure. Deutsch. Arch. f. klin. Med. 93, 331. 1908. 
einem Schweine und an einem Hahn festgestellt hatten, gingen sie zu den Versuchen an Menschen über. Sie führten zunächst solche an sich selbst aus und wiederholten dieselben dann an anderen gesunden Personen. Die Einzeldosen schwankten von $0,25-1 \mathrm{~g}$, die höchste Tagesdosis betrug $5 \mathrm{~g}$.

Das wesentlichste Ergebnis dieser Versuche war, daß kurze Zeit nach Einnahme der Substanz der Harn mehr oder weniger trübe gelassen wurde, ohne daß sich Beschwerden von seiten der Harnorgane zeigten. Die Trübung des Harns löste sich beim Zusatz von Alkalien, verschwand, wenn der Harn erwärmt wurde. Ließ man den Harn bei Zimmertemperatur einige Zeit stehen, so nahm die Trübung meist erheblich zu. Das sich beim Stehen des trüben Harns bildende Sediment hatte vielfach eine leicht rötliche Farbe und löste sich beim Erwärmen vollkommen auf, erschien aber beim Abkühlen des Harns wieder.

Die mikroskopische Untersuchung des trüben Harns hatte das Vorhandensein von rundlichen Gebilden ergeben, die zuweilen eine radiäre Streifung zeigten und im polarisierten Lichte betrachtet sich als doppelt lichtbrechend erwiesen. Sie zeigten sowohl in morphologischer Beziehung als auch in ihrem Verhalten zu chemischen Agentien eine weitgehende Übereinstimmung mit den Ablagerungen von harnsauren Salzen, wie sie Ebstein und $\mathrm{N}$ i cola i e $\mathrm{r}^{1}$ ) bei Kaninchen nach Einverleibung von Harnsäure in den Nieren und im Harn beobachtet und beschrieben haben.

Der in dem frisch entleerten trüben Harne enthaltene Niederschlag gab die Murexidreaktion und so sprach alles dafür, daß die Trübung des Harns nach Einnahme von Phenylcinchoninsäure durch die Ausscheidung harnsaurer Salze bedingt sei. Daß das Ausfallen derselben im frisch gelassenen Harne nicht etwa durch abnorme Löslichkeitsverhältnisse bedingt sei, sondern durch einen vermehrten Gehalt des Harns an Harnsäure, baben die weiteren Untersuchungen von Ni col a i r und Dohrn ergeben.

Die genannten Autoren fanden nach Verabreichung von Phenylcinchoninsäure eine Vermehrung der Harnsäureausscheidung von 78.3-331.1 Proz.

Nach Aussetzen der Phenylcinchoninsäure sinkt der Harnsäurewert unter die Norm und steigt erst allmäblich wieder zu den normalen Werten an.

1) Ebstein und Nicolaier: Über die Ausscheidung von Harnsäure durch die Nieren. Virchows Arch. 143. 337. 1896. 
Ueber die Beeinflussung des Purinstoffwechsels durch Phenylcinchoninsäure. 179

Die Wirkungsweise der Phenylcinchoninsäure auf die Ausscheidung der Harnsäure unterscheidet sich in Bezug auf zeitlichen Verlauf, Intensität und Ursache von der ähnlichen Wirkung der Salicylpräparate und ist mit dieser gewiß nicht zu identifizieren.

$\mathrm{N}$ i c o la i er und D o h r n haben versucht, eine Erklärung für diese eigenartige Wirkung der Phenylcinchoninsäure zu finden:

Zunächst konnten sie feststellen, daß es sich hier um eine Vermehrung der endogenen Harnsäure handle, da das gleiche Phänomen auch bei purinfreier Diät auftrat.

Daß der Überschuß an Harnsäure aus der verabreichten Substanz selbst entstehe, erscheint nach der Konstitution der Phenylcinchoninsäure, die überdies partiell unverändert im Harn wieder erscheint, ausgeschlossen.

Die Überproduktion an Harnsäure scheint nach Ansicht $\mathrm{Ni}$ c olaiers und Dohrns eine toxische Wirkung von Phenylcinchoninsäure zu sein und zwar auf diejenigen Faktoren, die bei der Harnsäurebildung in Betracht kommen.

Ob die gesteigerte Bildung der endogenen Harnsäure durch einen vermehrten Zellzerfall, oder durch vermehrten Auf- und Abbau der Zellkernsubstanzen oder endlich durch eine Beschleunigung der enzymatischen Oxydation der in den Muskeln gebildeten Purinbasen, insbesondere des Hypoxanthins, bedingt ist, lieb sich noch nicht entscheiden, doch halten $\mathrm{Nicol}$ a i er und $\mathrm{D}$ o h r n die letztgenannte Möglichkeit für die wahrscheinlichste Ursache des Phänomens. Daß die Harnsäurevermehrung durch Störung der Harnsäureoxydation bedingt sei, erscheint den Autoren unwahrscheinlich, da ein diesbezüglicher Versuch mit Rinderniere negativ ausfiel.

Am Schlusse ihrer interessanten Mitteilungen geben $\mathrm{N}$ i c o l a i e r u nd D o brn die Anregung, die Wirkung der Phenylcinchoninsäure auch in pathologischen Fällen, bei Störungen des Purinstoffwechsels zu prüfen, da die bedeutende Vermehrung der Harnsäureausscheidung durch Phenylcinchoninsäure vielleicht auch eine therapeutische Verwendung dieser Substanz ermöglichen könnte.

In diesem Sinne haben seither Tsehernikow und Magat $\left.{ }^{1}\right)$ an einer Reihe von Patienten Versuche angestellt und kamen zu folgenden Resultaten :

1) Tschernikow und Magat: Zur Frage des Einflusses der Phenylcinchoninsäure (Atophan) auf die Harnsäureausscheidung bei mit podagrischer Diathese und mit Rheumatismus behafteten Kranken. Charkower mediz. Journal. 1910. (Durch frdl. Vermittlung der Firma Schering in Übersetzung zugestellt.) 
Phenylcinchoninsäure bedingt stets eine Vermehrung der Harnsäureausscheidung, ohne Rücksicht auf die Diät der Patienten. Nach wiederholter täglicher Verabreichung der Substanz tritt eine Grenze der Wirkung ein, nach der eine Steigerung der Harnsäureausscheidung trotz fortgesetztem Gebrauch der Säure nicht mehr beobachtet wurde. Manchmal wurde gleichzeitig Steigerung der Diurese bemerkt. Bei keinem der Patienten bis auf einen mit Fettherz, wurde irgendwelche subjektive und objektive Nebenerscheinung beobachtet.

Bei Personen mit harnsaurer. Diathese wurde unmittelbar nach der Verabreichung der Phenylcinchoninsäure Besserung des objektiven und subjektiven Zustandes beobachtet, die in der Mehrzahl der Fälle ziemlich lange anbielt.

In Fällen von akutem und exazerbiertem Gelenkrheumatismus wurde ein auffallend rascher Effekt erzielt, nicht dagegen bei chronischem Rheumatismus.

I.

Die bisher vorliegenden Untersuchangen über die Wirkung der Phenylcinchoninsäure haben ergeben, daß dieser Substanz eine spezifische Wirkung auf den Purinstoffwechsel zukommt. Da eine Erklärung derselben bisher noch nicht gegeben war, so stand zu erwarten, durch das Experiment hierüber Aufschluß zu erhalten, vielleicht auch neue Gesichtspuukte in der Harnsäurefrage zu gewinnen.

$\mathrm{Zu}$ unseren Versuchen hatte uns die "Chemische Fabrik auf Aktien (vorm. E. Schering)" in Berlin die Phenylcinchoninsäure, die sie mit dem Namen „A tophan" bezeichnet, zur Verfügung gestellt und es sei ihr hierfür auch an dieser Stelle der beste Dank ausgesprochen.

$\mathrm{Nicolai}$ er und Dohrn haben es zwar schon versucht, bei einem Versuche an einem Schweine die Beeinflussung der Harnsäureausscheidung durch Phenyleinchoninsäure zu studieren, doch ist dieser Versuch mangels an quantitativen Allantoinbestimmungen unvollkommen; wir wissen heute durch die Arbeiten Wi echow sk is ${ }^{1}$ )

1) Wiechowski: Die Bedeutung des Allantoins im Harnsäurestoffwechsel, Hofmeisters Beiträge XI. 109. 1907.

Wiechowski: Über die Zersetzlichkeit der Harnsäure im menschlichen Organismus. Areh. f. exp. Path. u. Pharmak. 60. 185. 1909.

Wiechowski: Das Vorhandensein von Allantoin im normalen Menschenharn und seine Bedeutung für die Beurteilung des menschlieben Harnsäurestoffwechsels. Biochem. Zeitschrift 19. 368. 1909.

Wiechowski: Das Schicksal intermediärer Harnsäure beim Menschen und der Allantoingehalt des mensehlichen Harns; nebst Bemerkungen über Nachweis und Zersetzlichkeit des Allantoins. Biochem. Zeitschrift 25. 431. 1910. 
über den Purinstoffwechsel, daß zwischen Mensch und den übrigen Säugetieren hinsichtlich des Harnsäureschicksals eine scharfe Grenze gezogen werden muB. Wiechows ki kam in dieser Frage auf Grund seiner eingehenden Untersuchungen zu dem Schlusse, daß beim Menschen die Harnsäure das Endprodukt des Purinstoffwechsels darstellt und nur ein ganz kleiner Bruchteil als Allantoin zur Ausscheidung gelangt, während die übrigen Säugetiere Harnsäure woiter oxydieren können, so daß bei diesen Allantoin als Endprodukt des Purinstoffwhsls angesehen werden mulb, während hier Harnsäure nur in geringer Menge ausgeschieden wird.

Wenn nun beobachtet wurde, daß Phenylcinchoninsäure beim Menschen eine Vermehrung der Harnsäureausscheidung bedingt, so sollte dem bei Säugetieren eine vermehrte Allantoinausscheidung: entsprechen, vorausgesetzt, daß die Substanz bei beiden Organismen gleichartige Wirkung entfaltet.

Bevor wir nun derartige experimentelle Studien in Angriff nahmen, wollten wir uns von der Wirkung der Phenylcinchoninsäure am Menschen überzeugen.

Ich habe zu diesem Zwecke mehrere Versuche an mir selbst durchgeführt, dabei meistens bei purinfreier Diät.

Die Harnausscheidung gestaltete sich am Versuchstage, hinsichtlich der Harnfarbe, der Trübung usw. in ähnlicher Weise, wie sie Nicolaier und Dohrn beschrieben haben.

Die näheren Details dieser Versuche sind aus Tabelle I, VI, VII und VIII ersichtlich.

Tabelle I.

Versuchsperson: Verfasser. Gewicht $61 \mathrm{~kg}$.

\begin{tabular}{|c|c|c|c|c|c|}
\hline \multirow[t]{2}{*}{ Datum } & \multirow{2}{*}{$\begin{array}{c}\text { Harnmenge } \\
\text { in } \mathrm{ccm}\end{array}$} & \multirow{2}{*}{$\begin{array}{l}\text { Gesamt U } \\
\text { in } g\end{array}$} & \multicolumn{2}{|c|}{$\begin{array}{l}\text { Verhältnis der } \overline{\mathrm{U}} \\
\text { zum Normaltag }\end{array}$} & \multirow[t]{2}{*}{ Anmerkung } \\
\hline & & & in $\mathrm{g}$ & in $\%$ & \\
\hline 13.-15. VI. & - & - & - & 一 & Purinfreie Diät \\
\hline 16. V1. & 1230 & 0,3690 & - & 一 & dtto. \\
\hline 17. VI. & 1200 & 0,7470 & $+0,3780$ & $+102,27$ & $\begin{array}{l}\text { dtto. und } \\
\text { 3 mal } 0,5 \text { g Phenyl- } \\
\text { cinchoninsäure }\end{array}$ \\
\hline 18. VI. & 1230 & 0,3295 & $-0,0395$ & $-10,70$ & Purinfreie Diät \\
\hline
\end{tabular}

Der in Tabelle I mitgeteilte Versuch zeigt zunächst in Bestätigung der Befunde Nic o la iers und Doh rns ein Ansteigen der Harnsäureausscheidung um 102 Proz. am Versuchstage und ein Sinken derselben um fast 11 Proz. unter die Norm am Nachtage. 
An den beiden Tagen wurde auch eine Allantoinbestimmung in je 11 Harn nach der Methode Wi e ch ow sk is vorgenommen. Es gelang an beiden Tagen, wie sonst in der Norm, nur ganz geringe Mengen Allantoins in Kristallen zu erhalten, woraus hervorgeht, daß die bedeutende Änderung der $\overline{\mathrm{U}}$-Ausscheidung die Allantoinausscheidung unbeeinflußt ließ.

Es wurde weiters an Hunden und Kaninchen der Einfluß der Phenylcinchoninsäure auf die Allantoin- und Harnsäureausscheidung festgestellt.

Tabelle II zeigt vorerst die Resultate derartiger an Kaninchen durchgeführter Versuche.

Tabelle II.

Kaninchen 1820 g.

\begin{tabular}{|c|c|c|c|}
\hline Datum & $\begin{array}{c}\text { Harnmenge } \\
\text { in } \mathrm{ccm}\end{array}$ & $\begin{array}{l}\text { Allantoin } \\
\text { in } \mathrm{g}\end{array}$ & Anmerkung \\
\hline 6. V. & 20 & 0,0906 & \\
\hline 7. $\%$ & 40 & 0,0960 & \\
\hline 8. $n$ & 75 & 0,0869 & $\begin{array}{l}0,5 \mathrm{~g} \text { Phenylcinchoninsäure in Natr. } \\
\text { carb. gelöst subkutan }\end{array}$ \\
\hline 9. & 40 & 0,0537 & \\
\hline 12. & 45 & 0,0620 & $0,5 \mathrm{~g}$ Phenyleinchoninsä̈ure per os \\
\hline 13." & 45 & 0,0276 & \\
\hline
\end{tabular}

Der in Tabelle II mitgeteilte Versuch am Kaninchen fiel gegen unsere Erwartung aus; denn die Zufuhr von Phenylcinchoninsäure hatte nicht nur keine Vermehrung der Allantoinausscheidung zur Folge, sondern eine bedeutende Abnahme derselben. Da sich bei den Versuchen am Kaninchen einige bisher noch nicht aufgeklärte methodische .Schwierigkeiten zeigten und sich auf Grund der Versuche $\mathrm{W}$ i echowskis Hunde ebenso wie Kaninchen hinsichtlich des Purinstoffwechsels verhalten, so wurden zu den weiteren Versuchen stets Hündinnen verwendet; dieselben waren 8-14 Tage vor dem Versuche kolpotomiert worden, so daß der Harn durch Katheterisieren ohne Schwierigkeit quantitativ gewonnen werden konnte. Die Resultate derartiger Versuche bringen die Tabellen III-V.

Das wesentlichste Ergebnis der Versuche am Hunde ist eine Bestätigung des Resultates beim Kaninchen. Die Allantoinauss c heidung, die an den Normaltagen ziemlich konstant ist, wird nach Injektion von Phenylcinchoninsäure oder nach oraler Zufuhr der Substanz bedeutend beeinträchtigt, bisweilen verschwindet sie bis auf Spuren. 
Ueber die Beeinflussung des Purinstoffwechsels durch Phenylcinchoninsäure. 183

Tabelle III.

Weißer Spitz $8040 \mathrm{~g}$.

\begin{tabular}{|c|c|c|c|c|}
\hline Datum & $\begin{array}{l}\text { Harnmenge } \\
\text { in ccm }\end{array}$ & $\begin{array}{l}\text { Allantoin } \\
\text { in } g\end{array}$ & $\begin{array}{l}\text { Harnsäure } \\
\text { in } g\end{array}$ & Anmerkung \\
\hline 18. V. & 300 & 0,3055 & 0,0119 & \\
\hline 19. " & 150 & 0,3031 & - & \\
\hline 20. & 330 & 0,2188 & 0,0969 & $\begin{array}{l}1 \mathrm{~g} \text { Phenylcinchoninsäure in } \\
\text { Natr. carb. gelöst subkutan }\end{array}$ \\
\hline 21. $"$ & 300 & 0,2196 & - & \\
\hline
\end{tabular}

Tabelle IV.

Hündin $5100 \mathrm{~g}$.

\begin{tabular}{|c|c|c|c|c|}
\hline Datum & $\begin{array}{l}\text { Harnmenge } \\
\text { in cem }\end{array}$ & $\begin{array}{l}\text { Allantoin } \\
\text { in } g\end{array}$ & $\overline{\mathrm{U}}$ in $\mathbf{g}$ & Anmerkung \\
\hline 20. VI. & 210 & 0,20 & 0,0257 & \\
\hline 21. & 210 & 0,20 & 0,0310 & \\
\hline 22." & 160 & 0,19 & 0,0210 & $\begin{array}{l}\text { Futter entzogen. Wasser } \\
\text { ad libitum }\end{array}$ \\
\hline 23. " & 80 & 0,18 & 0,0140 & \\
\hline 24." & 85 & 0,157 & 0,0349 & $\begin{array}{l}\text { 0,5 g Phenylcinchoninsäure } \\
\text { subkutan }\end{array}$ \\
\hline 25. $"$ & 110 & 0,143 & 0,0230 & \\
\hline
\end{tabular}

Tabelle V.

Hündin $4500 \mathrm{~g}$.

\begin{tabular}{c|c|c|c|c}
\hline Datum & $\begin{array}{c}\text { Harnmenge } \\
\text { in ccm }\end{array}$ & $\begin{array}{c}\text { Allantoin } \\
\text { in } \mathrm{g}\end{array}$ & U in $\mathrm{g}$ & Anmerkung \\
\hline $\mathrm{7.} \mathrm{X}$. & 250 & 0,2200 & 0,0355 & \\
8. & 300 & 0,1900 & 0,0286 & \\
$9 . \%$ & 325 & 0,0011 & 0,0324 & $\begin{array}{c}0,5 \mathrm{~g} \text { Phenylcinchoninsäure } \\
\text { subkutan }\end{array}$ \\
$10 .$, & 300 & 0,2240 & 0,0217 &
\end{tabular}

Bei dem Versuche, der in Tabelle III mitgeteilt ist, wurde nach Zufuhr der Substanz beobachtet, daß der Hund weniger fraß. Obwohl bereits durch die Versuche Wi e chowskis (l. c.) nachgewiesen wurde, daß der Hunger auf die Allantoinausscheidung ohne Einfluß ist, habe ich doch den nächsten Versuch (Tabelle IV) am Hungertier durchgeführt. Das Resultat war das gleiche wie im ersten Versuch. 
Da das Allantoin im Hundeharn ein Oxydationsprodukt der Harnsäure ist, so war es noch notwendig, die Ausscheidung der Harnsäure zu bestimmen. Ist die Herabsetzung der Allantoinausscheidung durch eine Störung der Harnsäureoxydation bedingt, so muß gleichzeitig ein Ansteigen der Harnsäureausscheidung erfolgen. Dies ist nun tatsächlich in allen Versuchen der Fall.

Besonders deutlich zeigt sich dies in dem in Tabelle III mitgeteilten Versuche.

Wir finden dort am Normaltage: $0.0119 \mathrm{~g} \mathrm{U}$ u. $0.3031 \mathrm{~g}$ Allantoin am Versuchstage: $0.0903 \mathrm{~g} \overline{\mathrm{U}}$ u. $0.2188 \mathrm{~g}$ "

Die Harnsäure des Versuchstages wurde in schönen Kristallen gewogen, die frei von Verunreinigungen waren.

$0.0843 \mathrm{~g}$ Allantoin entsprechen $0.0903 \mathrm{~g}$ U.

Nach Abzug der am Normaltage ausgeschiedenen Harnsäure bleibt ein Rest von $+0.0850 \mathrm{~g}$ U.

Das Minus an Allantoin ist also nahezu vollständig als Harnsäure ausgeschieden worden.

Da wir in den folgenden Versuchen stets parallel mit der Abnabme der Allantoinausscheidung ein Ansteigen der Harnsäureausscheidung beobachteten, wenn auch nicht mit solcher Deutlichkeit, wie in dem in Tab. III mitgeteilten Versuche, so können wir doch den Schluß daraus ziehen, daß die vermehrte Ausscheidung der Harnsäure beim Hunde nach Phenylcinchoninsäureverfütterung durch eine Störung der Harnsäureoxydation zu Allantoin bedingt sei.

Nun haben $\mathrm{Nicolaier}$ und Dobrn bereits am Schweine einen derartigen Versuch durchgeführt und fanden in Übereinstimmung mit ihren Erfahrungen am Menschen eine Vermehrung der ausgeschiedenen Harnsäure. Daß sich das Schwein bezüglich seines Purinstoffwechsels ebenso verhält, wie die übrigen Säugetiere, geht aus den Versuchen Schittenhelms ${ }^{1}$ ) hervor und wir finden auch im Versuche Nicolaiers und Dohrns nur geringe. Harnsäurewerte. Leider ist in dem genannten Versuche die Allantoinausscheidung unberücksichtigt geblieben, doch sind darin gewisse Anhaltspunkte, daß auch hier ebenso wie in den von mir mitgeteilten Versuchen am Hunde die vermehrite Harnsäureausscheidung durch eine Störung ihrer Oxydation zu Allantoin bedingt ist. Nicola i e r u d. Doh r n beobachteten bei dem genannten Versuche, daß die Phenylcinchoninsäure auf die.Elimination

1) Schittenhelm: Über den Nukleinstoffwechsel des Schweines. Zeitschr. f. phys. Chem. 66. 53. 1910. 
des Gesamtstickstoffes keinen Einfluß nahm, während die Stickstoffzahl der Purinbasen am Versuchstage in die Höhe ging. Da der normalerweise als Allantoin ausgeschiedene Stickstoff mit dem $\stackrel{+}{\mathrm{U}-S t i c k-}$ stoff mitbestimmt wird, so können wir auf Grund obiger Beobachtung annehmen, daß hier [nur eine Verschiebung des Stickstoffwertes zugunsten der Purinbasen, stattgefunden hat, bedingt durch deren gestörte Oxydation.

Die Beeinflussung des urikolytischen Fermentes durch chemische Agentien läßjt sich auch in vitro durch einen Oxydationsversuch von Harnsäure durch geeignete Organpulver nachweisen.

In dieser Richtung haben Nicolaier und Dobrn bereits einen Versuch mit Rinderniere angestellt, kamen aber zu einem negativen Resultat. Ich habe derartige Versuche sowohl mit Hundeleber als auch mit Rinderniere wiederholt und kam zu gleichen Ergebnissen. Eine Störung der Oxydation der Harnsäure durch überlebende Organe findet bei Gegenwart von Phenylcinchoninsäure nicht statt. Es spricht dieses Resultat jedoch keineswegs dagegen, daß eine solche Störung in vivo vor sich gehe; denn $\mathrm{Nicol}$ a ier und D o h r n weisen bei einer andern Gelegenheit darauf hin, daß wahrscheinlich die Phenylcinchoninsäure nicht als solche, sondern in Form bestimmter Abbauprodukte ihre spezifische Wirkung auf den Purinstoffwechsel entfalte. Dies würde auch erklären, daß die im lebenden Organismus beobachtete Wirkung der Phenylcinchoninsäure nicht auch bei Oxydationsversuchen mit Organen zur Beobachtung gelangt.

Da in den bisher mitgeteilten Versuchen am Kaninchen und Hunde nur eine Abnahme der Allantoinausscheidung beobachtet wurde, gleichzeitig damit eine Zunahme der Harnsäureausscheidung, so wurde ein weiterer Versuch durchgeführt, bei dem neben Allantoin und $\bar{U}$ auch der Purinbasenstickstoff nach $\mathrm{Ca}$ m er e r bestimmt wurde.

\section{Versueh 6.}

Eine $6150 \mathrm{~g}$ schwere Hündin schied aus am 20. I. $325 \mathrm{cem}$ Harn, $0.13 \mathrm{~g}$ Allantoin, $0.0058 \mathrm{~g}$ Harnsäure und $0.0036 \mathrm{~g}$ Purinbasen $\mathrm{N}$.

Am 21. I. $400 \mathrm{ccm}$ Harn, $0.19 \mathrm{~g}$ Allantoin, $0.0074 \mathrm{~g} \mathrm{U}, 0.0046 \mathrm{~g}$ Purinbasen-N.

Nun erhielt sie $1 \mathrm{~g}$ Phenylcinchoninsäure in Natr. carb. gelöst subkutan injiziert.

Am folgenden Tag betrug die Ausscheidung $425 \mathrm{ccm}$ Harn, $0.0320 \mathrm{~g} \overline{\mathrm{U}}$ und $0.003 \mathrm{~g}$ Purinbasen-N. Die Allantoinausscheidung war zu gering um bestimmt werden, zu können, somit sicher bedeutend herabgesetzt. 
Wenn wir nun die Resultate dieser Versuche überblicken, so fragt es sich zunächst, ob wir aus denselben Anhaltspunkte für die Erklärung der spezifischen Wirkung der Phenylcinchoninsäure auf den Purinstoffwechsel des Menschen gewonnen haben.

Unter Beziehung auf die Ergebnisse der Untersuchungen Wiechowskis müssen wir dies negieren; denn wenn wir eine Störung der Harnsäureoxydation als Ursache ihrer vermehrten Ausscheidung beim Hunde, Kaninchen, Schweine usw, erklärlich finden, so ist dies beim Menschen ausgeschlossen. Wi e chow ski hat es zur Genüge bewiesen, daß wir beim Menschen in der Harnsäure das Endprodukt des Purinstoffwechsels vor uns haben und daß von einer Oxydation der Harnsäure beim Menschen überhaupt nicht gesprochen werden kann.

Entgegengesetzte Anschauungen und Einwände wußte Wi ech ow ski ebenfalls zu entkräften. Die Wirkung der Phenylcinchoninsäure auf den menschlichen Purinstoffwechsel muß eine ganz andere sein als beim Hunde, Schweine und anderen Säugetieren; wieder geht aus den bisher mitgeteilten Versuchen überzengend hervor, daß wir zwischen dem $\mathrm{Purinst}$ offwechsel des Menschen und der übrigen Säugetiere strenge Grenzen ziehen müssen.

II.

Die experimentellen Untersuchungen an Säugetieren hatten so zur Erklärung der Phenylcinchoninsäurewirkung beim Menschen nichts beigetragen. Es wurden daher zum Studium dieser Substanz noch Versuche am Huhn ausgeführt.

Der Vogelorganismus hat mit dem menschlichen wenigstens das eine gemeinsam, daß bei ihm die Harnsäure als Endprodukt des Purinstoffwechsels angesehen werden muß. Hinsichtlich der Harnsäurebildung steht jedenfalls die Synthese im Vordergrunde, während beim Säugetier eine solche mit voller Sicherheit noch nicht erwiesen ist. Daß jedoch auch der Vogel Harnsäure durch Oxydation von Purinbasen bildet, ist durch die Versuche $M$ inkowskis und v. $\mathrm{M} \mathrm{a} \mathrm{c} \mathrm{h}^{1}$ ) nachgewiesen worden.

Es war also zu erwarten, daß beim Vogel Phenylcinchoninsäure ebenfalls eine Vermehrung der Harnsäure bewirkt, wenn die beobachtete Vermehrung beim Menschen durch eine Beeinflussung der Purinoxydation oder der möglicherweise vorhandenen Harnsäuresynthese bedingt ist.

1) v. Mach: Über die Umwandlung von Hypoxanthin in Harnsäure im Organismus der Vögel. Arch. f. exp. Path. u. Pharmak. 24. 389. 1888. 
Die Versuche wurden an Hähnen ausgeführt, die in einem Zwangskäfig gehalten wurden. Harn und Fäkalien wurden gemeinsam unter Znsatz von Karbolalkohol aufgefangen, dann mit Gips zu einer homogenen Masse verrieben und diese bei ca. $55^{\circ}$ getrocknet. Man erhält auf diese Weise ein trockenes Pulver, in dem alle Bestandteile gleichmäBig verteilt sind und das für die jeweiligen Untersuchungen beliebig lange aufbewahrt werden kann. Zur Harnsäurebestimmung wurde ein aliquoter Teil mit heißem, schwach alkalischem Wasser solange extrahiert, bis das Filtrat mit ammoniakalischer Silberlösung nicht mehr reagierte. In den vereinigten Filtraten wurde die Harnsäure nach Ludwig-Salkowski bestimmt.

Im folgenden sind die Resultate dieser Versuche mitgeteilt:

\section{Versuch 7 .}

Ein Hahn $(1380 \mathrm{~g})$ erhält, vom 2. XII. angefangen, täglich $50 \mathrm{~g}$ Mais als Futter, Wasser ad libitum.

Er bleibt während des Versuches im Gewichte gleich.

5. XII. $=1.2800 \mathrm{~g} \overline{\mathrm{U}}$ pro die.

6. XII. $=1.2620 \mathrm{~g} \mathrm{U}$

Nun erhält der Hahn $1 \mathrm{~g}$ Phenylcinchoninsäure in Brotpillen der Nahrung zugesetzt.

Es trat daraufhin reichliche Diurese ein. Die Fäkalien zeigten gelbliche Auflagerungen.

7. XII. $=0.3986$ g U pro die.

Am 8. und 9. XII. waren nur geringe Mengen von Harnsäure nachweisbar.

16. XII. $=1.4013 \mathrm{~g} \mathrm{U}$ pro die.

Das Resultat dieses ersten Versuches war wiederum gegen alle Erwartung ausgefallen; denn statt der erwarteten Steigerung der Harnsäureausscheidung unter dem Einfluß von Phenylcinchoninsäure war dieselbe stark herabgesetzt worden.

Da, wie bereits erwähnt, die Oxydation der Purinbasen zur Harnsäure beim Vogel gegenüber deren synthetischen Bildung zurücktritt, so war zu vermuten, daß im vorliegenden Falle die Synthese der Harnsäure eine Störung erlitten hatte. Es war an die Möglichkeit zu denken, daß an den Tagen, wo nur wenig Harnsäure ausgeschieden wurde, vielleicht die Ausscheidung des Harnstoffs eine Steigerung erfahren hatte.

Es wurde, um dies zu erfahren, ein Teil der Fäkalien des Normaltags sowie der Versuchstage nach vorhergehender Vertreibung des Ammoniaks durch Magnesia usta mit Äther-Alkohol (1:3) extrahiert, die Filtrate im Vacuum zur Trockene eingedampft, dann in wenig angesäuertem Wasser aufgenommen und der N-Gehalt des Rückstands nach Kjeldahl bestimmt. 
Rückstand des Äther-Alkoholextraktes:

5. XII. $0.0235 \mathrm{~g} \mathrm{~N}=0.0504 \mathrm{~g} \stackrel{+}{\mathrm{U}}$.

7. XII. $0.0469 \mathrm{~g} \mathrm{~N}=0.1006 \mathrm{~g} \stackrel{+}{\mathrm{U}}$.

8. XII. $0.0812 \mathrm{~g} \mathrm{~N}=0.1714 \mathrm{~g} \stackrel{+}{\mathrm{U}}$.

An den Tagen, an denen die U-Ausscheidung herabgesetzt war, gingen die $\stackrel{+}{\mathrm{U}}-$ Werte in die Höhe, so daß eine Störung der Harnsäuresynthese angenommen werden muß. Rechnet man das Plus an $\stackrel{+}{\mathrm{U}}$ in $\overline{\mathrm{U}} \mathrm{um}$, so findet man, daß dem Normaltag gegenüber ein beträchtliches $\overline{\mathrm{U}}$-Defizit bestand, so daß auch eine Minderung des N-Zerfalls vorhanden gewesen sein $m u ß$.

Versuch 8.

In einem 2. Versuch schied ein Hahn an 2 Normaltagen $2.8573 \mathrm{~g}$ U aus, an den zwei Tagen nach Zufubr von Phenylcinchoninsäure $2.4239 \mathrm{~g} \mathrm{U}$. Die Harnstoffausscheidung hatte keine nennenswerte Änderung erfahren.

Die zweite Versuchstype hat somit ergeben, daß auch der Purinstoffwechsel des Vogels durch Phenylcinchoninsäure eine Störung erfährt; die Störung ist jedoch ganz anderer Art als beim Menschen und bei den Säugetieren. Auffallenderweise äußert sich in allen drei Fällen ein Einfluß der genannten Substanz auf den Purinstoffwechsel, doch immer in verschiedener Weise.

III.

Die Versuche am Huhn konnten infolgedessen auch keine Erklärung bringen für die Wirkung der Substanz beim Menschen. Es mulbten daher die Versuche am Menschen wieder aufgenommen werden und zwar wurde nunmehr die Wirkung der Substanz auf den menschlichen Organismus in gewissen Einzelheiten studiert, ihr Einfluß auf die fermentative Tätigkeit menschlicber Organe, soweit dieșe für den Purinstoffwechsel in Betracht kommen, verfolgt.

Die Unzersetzlichkeit der Harnsäure im menschlichen Organismus ist einwandfrei nachgewiesen. Die vermebrte Ausscheidung von Harnsäure unter dem Einflusse der Phenylcinchoninsäurewirkung beim Menschen kann daher nicht als eine Störung einer normalen Harnsäureoxydation angesehen werden, wie wir dies für den Hund und andere Säugetiere nachgewiesen haben.

Da aber Schittenhelm und andere Autoren immer noch der Anschauung sind, daß auch im menschlichen Organismus ein Teil der Harnsäure oxydiert wird und zwar bis zu Harnstoff, so könnte 
nach dieser Auffassung auch im vorliegenden Falle die vermehrte Harnsäureausscheidung im Sinne einer gestörten Oxydation gedeutet werden. Um derartigen Einwänden zu begegnen, habe ich eine Reihe von Versucben an mir selbst ausgefübrt, bei denen auf eine genaue Stickstoffbilanz geachtet wurde. Um möglichst gleiche Bedingungen für den Versuch zu schaffen, habe ich während der ganzen Versuchszeit unter vollkommen gleichen Bedingungen gelebt, die gleiche Nabrung: (purinfrei) und die gleiche Flüssigkeitsmenge aufgenommen.

Bei dem in Tabelle VI mitgeteilten Versuche hielt ich folgende Diät ein:

Horgens: $250 \mathrm{ccm}$ Milch, eine Semmel.

Vormittags: zwei Semmeln, $30 \mathrm{~g}$ Butter.

Mittags: Mehlspeise aus $50 \mathrm{~g}$ Weizenmebl und zwei Eiern. $100 \mathrm{~g}$ Ko mpott, zwei Eier, $250 \mathrm{ccm}$ Milch, eine Semmel.

Nachmittags: zwei Semmeln, $30 \mathrm{~g}$ Butter.

Abends: Griesbrei aus $50 \mathrm{~g}$ Griesmehl, zwei Eier, eine Semmel, : $1 / 4 \mathrm{l}$ Milch, $1 / 4 \mathrm{~kg}$ Obst, zwei Semmeln mit Fett.

Die einzelnen Stickstofffraktionen wurden nach $\mathrm{Pf}$ aundler bzw. Kriiger und Schmidt bestimmt, die P2O5-Bestimmung erfolgte durch Urantitration. Harnsäurewerte nach Ludwig-Salkowski.

Mit der purinfreien Diät wurde am 16. X. begonnen mit der Harnuntersuchung am 18. X. Das Resultat des Versuches ist aus Tabelle VI ersichtlich.

Nach den Anschauungen von Frank und Schittenhelm ${ }^{1}$ ) wäre der Stickstoff der Harnsäure, die im menschlichen Organismus abgebaut wird, in der Harnstofffraktion zu finden. Würde die Vermehrung der Harnsäure als Folge der Pbenylcinchoninsäurewirkung durch Störung der Oxydation eines Teiles derselben zu Harnstoff bedingt sein, so müßte gleichzeitig eine Verschiebung des Stickstoffwertes von $\stackrel{\dot{\mathrm{U}}-\mathrm{N}}{\mathrm{zu}} \mathrm{PWS}-\mathrm{N}$ stattfinden and zwar zugunsten des letzteren.

In dem in Tabelle VI mitgeteilten Versuche ist wohl eine solche Verschiebung vorhanden; daß diese Zablen jedoch - namentlich was den Harnstoffstickstoff betrifft - innerhalb der physiologischen Schwankungen liegen und nicht die Folge der Phenyleinchoninsäurewirkung darstellen, dáfür spricht besonders der in Tabelle VII mitgeteilte Versuch, bei dem trotz einer bedeutenden Zunahme der Harnsäure nach Phenylcinchoninsäure der Harnstoffstickstoff keine Abnahme gefunden hat.

Bei dem in Tabelle VII mitgeteilteu Versuche wurde neben der purinfreien Kost auch Natr. lactic. eingenommen. Es war immer noch an die Möglichkeit zu denken, daß die Wirkung der Phenylcinchoninsäure darin bestehe, daß sie eine sonst nur in geringem

1) F. Frank u. A. Schittenhelm. Über die Umsetzung verfütterter Nukleinsäure beim normalen Menschen. Z. f. phys, Chem. 63. 243. 1909. 
Maße vorhandene synthetische Harnsäurebildung fördere. Als Komponente für diese Synthese könnte Milchsäure in Betracht kommen.

Die purinfreie Diät bei diesem Versuche war mit geringen Änderungen dieselbe wie im vorhergehenden Versuche und zwar vom 23.X. angefangen. Mit der Untersuchung der Harne wurde am 27. X. begonnen.

Die Resultate des Versuchs enthält Tabelle VII.

Wie aus diesem in Tabelle VII mitgeteilten Versuche hervorgeht, sehen wir hier wiederum ein Ansteigen des Harnsäurewertes nach Einnahme von Phenylcinchoninsäure und ein Absinken desselben unter die Norm am nachfolgenden Tage, ohne daiß dabei der Wert für den Harnstoffstickstoff eine Änderung in dem oben angedeuteten Sinne erfahren würde. Ein Einfluß der zugeführten Milchsäure auf die U-Ausscheidung war nicht zu bemerken.

Der in Tabelle VII angeführte Versuch spricht also nicht nur nicht für die Anschauungen Schittenhelms, er enthält sogar Anhaltspunkte, die gegen die Vorstellungen Schittenhelms vom Abbau der Harnsäure sprechen.

In obigem Versuche wurde die Harnsäureausscheidung von $0.44 \mathrm{~g}$ auf $0.77 \mathrm{~g}$ gesteigert, also um $0.33 \mathrm{~g}$. Da diese Vermehrung, wie später ausgeführt werden soll, wahrscheinlich durch einen gesteigerten Zerfall des U-bildenden Materials bedingt ist und die $\stackrel{+}{\mathrm{U}}$-Ausscheidung keine Vermehrung erfahren hat, so ist dadurch wiederum gezeigt, daß auch die endogen entstehende Harnsäure als solche ausgeschieden wird und keinen weiteren Abbau erfährt.

Eine Erklärung der Barnsäurevermehrung beim Menschen nach Phenylcinchoninsäure durch Störung der Oxydation erscheint also auch auf Grund dieser.Versuche ausgeschlossen.

Aus den vorstehenden Versuchen geht hervor, daß die Phenylcinchoninsäure keine gleichgültige Substanz ist, daß sie eingreifende Veränderungen im Purinstoffwechsel hervorruft und dies führt uns wieder zu der bereits von Nicolaier und Dohrn geäußerten Ansicht, daß die Überproduktion an Harnsäure als eine Art von tox ischer Wirkung der Phenylcinchoninsäure auf diejenigen Faktoren angesehen werden muß, die bei der Harnsäurebildung in Betracht kommen. Dabei erklären sich die Autoren das Absinken der Harnsäure nach der infolge von Phenylcinchoninsäuregebrauch eingetretenen Vermehrung in der Weise, daß der menschliche Körper, nachdem er größere Mengen harnsäurebildenden Materials verloren hat, mit dem noch vorhandenen spart, bis das Defizit ausgeglichen ist. 
Ueber die Beeinflussung des Purinstoffwechsels durch Phenylcinchoninsäure. 191

\begin{tabular}{|c|c|c|c|c|c|}
\hline & 完密寄 & & & 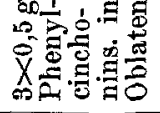 & \\
\hline & 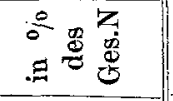 & $\begin{array}{l}\mathbb{N} \\
\infty \\
\infty \\
\infty\end{array}$ & $\begin{array}{l}8 \\
0 \\
\infty \\
\infty\end{array}$ & 焉 & 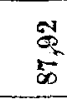 \\
\hline & $+\frac{7}{b}$ so & $\begin{array}{l}\bar{N} \\
\stackrel{-}{2}\end{array}$ & 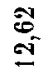 & $\stackrel{8}{\stackrel{0}{0}}$ & : \\
\hline & 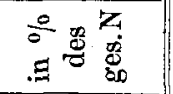 & 구 & $\Xi$ & $\underset{\Xi}{=}$ & $\stackrel{\tilde{g}}{\mathrm{~g}}$ \\
\hline & 竞它分 & $\underset{\Xi}{\stackrel{Z}{0}}$ & $\stackrel{\check{z}}{\sigma}$ & $\stackrel{\text { : }}{\stackrel{1}{0}}$ & $\frac{\pi}{6}$ \\
\hline & 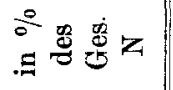 & $\begin{array}{l}50 \\
5 \\
\infty\end{array}$ & 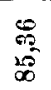 & F & $\begin{array}{l}\vec{D} \\
\infty \\
\infty \\
\infty\end{array}$ \\
\hline & 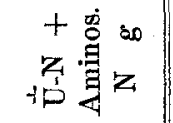 & $\underset{m}{79}$ & 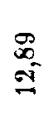 & $\stackrel{\infty}{\infty}$ & 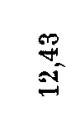 \\
\hline 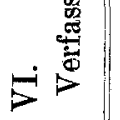 & 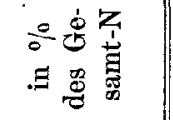 & 尊 & : & $\begin{array}{l}0 \\
\substack{10 \\
0} \\
0\end{array}$ & $\stackrel{\circ}{=}$ \\
\hline 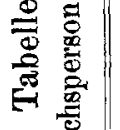 & 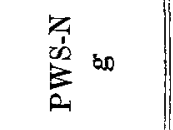 & $\stackrel{5}{\sigma}$ & $\overrightarrow{\text { ন্}}$ & 足 & $\stackrel{8}{-1}$ \\
\hline 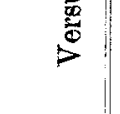 & 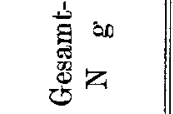 & 量 & $\frac{5}{15}$ & $\stackrel{9}{ \pm}$ & $\stackrel{8}{g}$ \\
\hline & $\begin{array}{l}\therefore \\
\therefore\end{array}$ & 1 & 1 & $+\stackrel{5}{\stackrel{5}{9}}$ & $1 \stackrel{\infty}{=}$ \\
\hline & 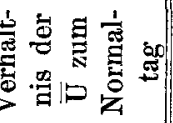 & 1 & 1 & $+\underset{\tilde{g}}{0}$ & $1 \frac{5}{50}$ \\
\hline & 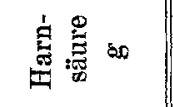 & 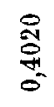 & 紫 & $\begin{array}{l}0 \\
\substack{0 \\
\infty \\
0 \\
0 \\
0}\end{array}$ & 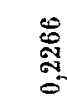 \\
\hline & 实 & $\stackrel{\infty}{\infty}$ & $\stackrel{\infty}{\infty}$ & $\stackrel{28}{\circ}$ & $\stackrel{\infty}{\circ}$ \\
\hline & 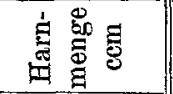 & $\stackrel{\Xi}{\Xi}$ & 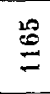 & 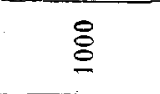 & 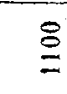 \\
\hline & 皇 & $\begin{array}{l}\dot{x} \\
\infty\end{array}$ & $\dot{4}$ & வें & $\stackrel{\dot{N}}{\dot{N}}$ \\
\hline
\end{tabular}


XII. E. Starkenstein.

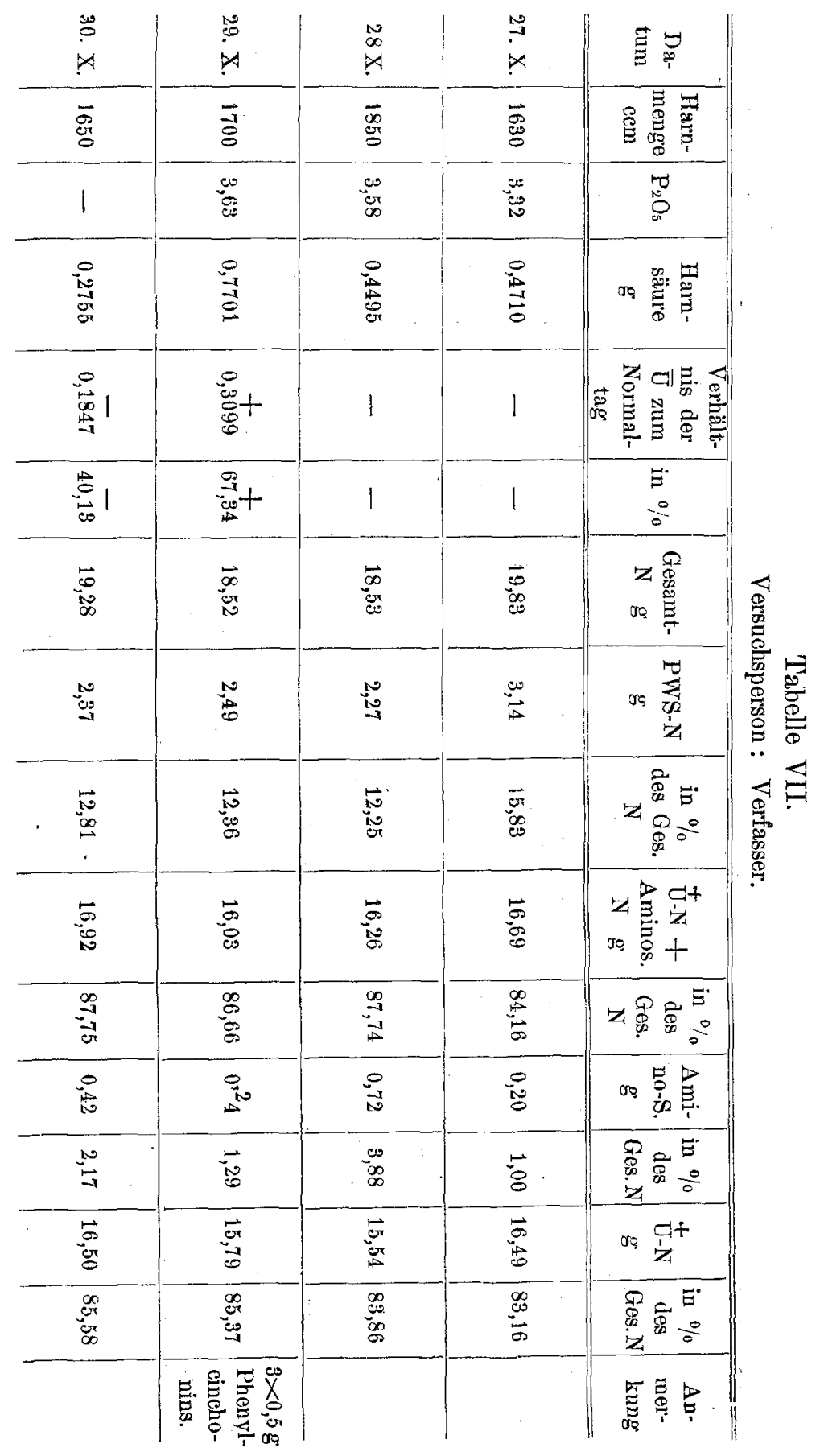


Als harnsäurebildendes Material kommen vor allem die Nucleoproteide in Betracht. In einer gesteigerten Ausscheidung, der Phosphorsäure wäre ein Indikator für einen 'gesteigerten Nucleinzerfall gegeben. Hierauf haben bereits $\mathrm{Nicolaier}$ und Dohrn geachtet und fanden während der Wirkung der Phenylcinchoninsäure keine vermehrte Phosphorsäureansseheidung.

$\mathrm{Zu}$ gleichen Resultaten kam ich in dem in Tabelle VI mitgeteilten Versuche, wo die Phosphorsäureausscheidung am Versuchstage sogar eine geringe, allerdings nicht nennenswerte Herabsetzung erfuhr, während im Versuche der Tabelle VII ein leichter Anstieg derselben beobachtet wurde.

$\mathrm{Zu}$ berücksichtigen ist dabei, dab wir entsprechend der vermehrten Harnsäurezahl nach der bekannten Zusammensetzung der Nucleoproteide nur einen geringen Anstieg der Phosphorsäure erwarten dürfen. Anderseits ist aber die Phosphorsäure der Nucleoproteide in organischer Bindung vorhanden und könnte bei gesteigertem Zellzerfall entweder als solche ausgeschieden oder retiniert und angesetzt werden. Was nun vorerst die Ausscheidung organischer Phosphate durch den Harn anlangt, so ist für einen eventuellen Nachweis derselben im Harne die entsprechende Methodik zu berücksichtigen. Bei den in den obigen Tabellen angefübrten Versuchen wurde die Phosphorsäure stets durch Urantitration bestimmt. Die Werte waren vor und nach Aufnahme der Phenylcinchoninsäure gut übereinstimmend. Auf Grund bereits früher von mir mitgeteilter Versuche über die Methodik der Phosphorsäurebestimmang im Harne ${ }^{1}$ ) wissen wir, daß der dureh Urantitration bestimmte Phosphorsäurewert nicht bloß anorganische Phosphorsäure angibt,", sondern auch häufig organische, so Glyzerinphosphorsäure oder die in den meisten Harnen vorkommende Inositphosphorsäure.

Wir wissen nicht, welcher Art die bier in Betracht kommende organische Phosphorsäure ist, können aber vermuten, daß es sich um eine Pentosephosphorsäure handelt ${ }^{2}$ ). Wir wissen auch von vornherein nicht, durch welche der gebräuchlichen Phosphorsäurebestimmungsmetboden dieselbe im Harne bestimmt werden kann; denn dies wird, wie in den übrigen Fällen, stets erst von den Eigensehaften der vorliegenden gepaarten Phosphorsäure abbängen.

Immerhin können wir, wenn wir in ein und demselben Harne die Phosphorsäure durch mebrere Methoden nebeneinander bestimmen, dafür sichere Anhaltspunkte gewinnen, ob überhaupt irgend-

1) Starkenstein: Die biologische Bedeutung der Inositphosphorsäure. Biochem. Zeitschrift 30. 55. 1910.

2) Neubergu. Brahm: Über die Inosinsäure. Biochem. Zeitschr. 5. 5̌60. 1908. 
eine organisch gebundene Phosphorsäure nach Phenylcinchoninsäureaufnahme in Harne vorbanden ist.

Es wurde daher in einem weiteren Versuche neben der Harnsäure auch die Phosphorsäure durch Urantitration, durch die Molybdänmethode vor und nach der Veraschung bestimmt.

Das Resultat des Versuches ist aus Tabelle VIII ersichtlich.

Tabelle VIII.

Versuchsperson: Verfasser. Konstante gemischte Kost.

\begin{tabular}{|c|c|c|c|c|c|c|}
\hline \multirow[b]{2}{*}{ Datum } & \multirow{2}{*}{$\begin{array}{l}\text { Harn- } \\
\text { menge } \\
\text { ccm }\end{array}$} & \multirow{2}{*}{$\begin{array}{l}\text { Harn- } \\
\text { säure } \\
\text { in } g\end{array}$} & \multicolumn{3}{|c|}{$\mathrm{P}_{2} \mathrm{O}_{5}$ bestimmt durch } & \multirow{2}{*}{$\begin{array}{c}\text { An- } \\
\text { merkung }\end{array}$} \\
\hline & & & $\begin{array}{l}\text { Uran- } \\
\text { titration }\end{array}$ & $\begin{array}{c}\text { Molybdän } \\
\text { direkt }\end{array}$ & $\begin{array}{l}\text { Molybdän } \mathbf{n} \text {. } \\
\text { d.Veraschung }\end{array}$ & \\
\hline 7. XII. & 1500 & 0,8235 & 3,2865 & 3,2010 & 3,3630 & \\
\hline 8. , & 1600 & 0,6784 & 3,5056 & 3,3440 & 3,5060 & $3 \mathrm{mal} 0,5 \mathrm{~g}$ \\
\hline 9. , , & 1920 & 1,1462 & 3,1304 & 2,9932 & $3,1.792$ & $\begin{array}{l}\text { Phenyl- } \\
\text { cinchonin- } \\
\text { säure }\end{array}$ \\
\hline 10. & 1650 & 0,6839 & - & - & - & \\
\hline
\end{tabular}

Der eben mitgeteilte Versuch zeigt, daß auch durch die genaue Methodik der Phosphorsäurebestimmung nicht mehr organisch gebundene nachzuweisen war, als in normalen Harnen vorhanden ist.

Es bleibt somit nur die Annahme übrig, daß bei dem möglicherweise unter dem Einflusse der Phenylcinchoninsäure eintretenden Nucleinzerfall, die freiwerdende organische Phosphorsäure retiniert und assimiliert wird.

Daß die Wirkung der Phenyloinchoninsäure beim Menschen darin besteht, daß sie das harnsäurebildende Material in irgendeiner spezifischen Weise beeinflußt, etwa derart, daß sie die Zellen rascher zum Zerfall bringt oder die Oxydation der freigewordenen Purine fördert, ist vorläufig die allein verbliebene mutmaßliche Erklärung. Es wurde noch versucht, für diese durch Fermenstudien mit menschlichen Organen unter dem Einflusse der Phenylcinchoninsäure einen experimentellen Beleg zu finden.

Ich habe das harnsäurebildende Vermögen der Menschenleber bei Gegenwart von Phenylcinchoninsäure geprüft. Die Versuche blieben erfolglos, selbst nach Zusatz von nucleinsaurem Natron.

Schließlich wäre noch an die Möglichkeit zu denken, die beobachtete Vermehrung der $\bar{U}$-Ausscheidung als ein Nierenphänomen zu deuten. Es könnte unter dem Einfluß dieser Substanz die Niere für $\bar{U}$ durchlässiger werden, ähnlich wie nach Phlorhidzin für Zucker. Dafür würden besonders die Versuche am Hunde sprechen; dort wäre die vermehrte U-Ausscheidung auf Kosten des Allantoins derart zu erklären, daß die Harnsäure so rasch aus ddem Körper eliminiert 
wird, daß sie nicht erst zu Allantoin oxydiert werden kann. Dieser Erklärungsmodus erscheint jedoch nicht”sehr wahrscheinlich. Zunächst fehlt in allen Versuchen eine gesteigerte Diurese, die eine vermehrte U-Ausschwemmung erklären würde, ferner erscheint es als überflüssig anzunehmen und das Problem gar nicht erscböpfend. daß die Niere, die schwankende Mengen von Harnsäure sekretorisch zu bewältigen vermag, plötzlich noch durchlässiger werden sollte.

Wir wissen, daß durch die Niere täglich einige deg. Harnsäure ausgeschieden werden. Nach reichlicher Zufuhr von Purinen oder nach direkter Harnsäureinjektion führt die Niere auch das Plus an Harnsäure ab; es kommt also normalerweise nicht erst zur Bildung von Harnsäuredepots. Das Plus von Ū pro die nach Phenylcinchoninsänre wäre auch durch die Hilfshypothese einer gesteigerten Durchlässigkeit der Niere gar nicht aufgeklärt.

Es ist gewiß kein Grund vorhanden, anzunehmen, daß durch die Phenylcinchoninsäure eine leichtere Elimination bereits vorhandener Harnsäure erfolgt, vielmehr spricht alles dafür, $d$ a $B$ u n t e r dem EinfluB der Substanz die zum Zerfall bestimmten Nucleoproteiderascher zum Abbaugebracht werden und auf diese Weise eine vermebrte Bildung der endogenen Harnsäure bedingen, dieam folgenden Tage durch ein Herabsinken der Harnsäureausscheidung unter die Norm wieder ausgeglichen wird.

Ni c o laier und D o h r n haben, wie bereits erwähnt, die Anregung gegeben, die Phenylcinchoninsäure auch bei Gichtikern zu prïfen und Tsehernik ow und $\mathrm{Magat}$ kamen, wie oben angeführt, dieser Anregung folgend, anscheinend zu einem guten Resultate 1 ).

Es fragt sich nun, ob wir bei unseren Untersuchungen Anhaltspunkte gewonnen haben, die für oder gegen die therapeutische Verwendung der Phenylcinchoninsäure oder des Atophans sprechen würden.

Die eine Erfahrung ist jedenfalls voranzustellen, daß Phenylcinchoninsäure sowohl beim Säugetiere als auch beim Vogel ein-

1) Anmerkung bei der Korrektur: Inzwischen sind hierhergehörige Mitteilungen von L. Heller und von Weintraud erschienen. L. Heller (Berl. Klin. Wochenschr. 526, 1911) hat Atophan bei 7 Fällen von akuter Gicht und bei 40 Fällen von Gelenksrheumatismus mit günstigem Erfolge angewendet. Leider fehlen dort Zahlen über die Harnsäureausscheidung. Wein trau d (Therapie der Gegenwart. März 1911) hat das Mittel seit 2 Jahren bei einer gröBeren Anzahl Gichtkranker Klinisch erprobt und es wie beim akuten Gichtanfall auch zur Beseitigung alter Harnsäureablagerungen erfolgreich gefunden. Genaue Bestimmungen ergaben sehr bedeutende Steigernngen der Harnsäureausscheidung, auch bei längere Zeit (durch 12 Tage) fortgesetztem Gebrauch. 
greifende Veränderungen des normalen Purinstoff wechsel herbeiführt und es muß als wahrscheinlich gelten, daß auch beim Menschen dieVermehrung der Harnsäure nach Zufuhr der Substanz untertoxischen Einflüssen steht.

Da jedoch selbst nach Zufuhr größerer Dosen weder eine objektive noch eine subjektive Anderung des Befindens eintritt, so ist die Frage zu diskutieren, ob das Atophan beim Gichtiker therapeutisch verwendet werden soll.

Die Erfahrung, daß Phenylcinchoninsäure die Harnsäurebildung steigert, würde jede Verwendung der Substanz beim Gichtiker a priori ausschließen.

Nun können wir uns aber recht wohl vorstellen, daß der Zellbestand des menschlichen Organismus derart gestaltet ist, daß stets ein Teil dem Untergang geweiht ist. Diese zum Zerfall bestimmten Nucleoproteide sind die Quellen für die frei werdenden Phosphorsäuren sowie für die endogene Harnsäure. Diese stellt bereits das Endprodukt der frei gewordenen Purinbasen dar und wird durch den Harn ausgeschieden.

Anders liegen die Verbältnisse bei der Gicht. Es sprechen zahlreiche Erfahrungen dafür, daß es sich hier um eine gestörte Ausscheidung, um eine pathologische Retention der Harnsäure im Organismus handelt. Von der endogenen Harnsäure wird in diesem Falle - bei sonstiger purinfreier Diät - nur ein kleiner Teil ausgeschieden, ein Teil jedoch bestimmten pathologischen Depots im Körper zugeführt

Wenn nun Phenylcinchoninsäure imstande ist, einerseits den Zerfall der hierzu prädestin i erten Nucleoproteide zu fördern, daß nur solche in Betracht kommen, dafür spricht die Abnahme der Harnsäureausscheidung $\mathrm{n} \mathrm{a} \mathrm{ch}$ der Phenylcinchoninsäurewirkung sowie bei protrahierter Darreichung der Substanz - anderseits unter dem Einfluß der Substanz die Harnsäureretention in den Gelenken, Knorpeln usw. unmöglich würde, so wäre dies für den Gichtiker gewiß von großem Werte.

Daß das Atophan beim Gichtiker eine derartige Wirkung ausübt, erscheint auf Grund der wenigen, bisher vorliegenden Angaben wahrscheinlich.

Jedenfalls regen die bisherigen Erfahrungen zu weiteren klinischen Versuchen an und erst eine größere Anzahl derselben wird ein endgültiges Urteil ermöglichen.

Ich selbst habe weitere Untersuchungen über die Wirkung der Phenylcinchoninsäure bei künstlich gichtisch gemachten Tieren in Angriff genommen, deren Mitteilung ich mir, da die Versuche längere Zeit in Anspruch nehmen, für eine spätere Zeit vorbebalte. 